\title{
Acoustic analog of electromagnetically induced transparency in periodic arrays of square rods
}

\author{
Fengming Liu, ${ }^{1}$ Manzhu Ke, ${ }^{1}$ Anqi Zhang, ${ }^{1}$ Weijia Wen, ${ }^{2}$ Jing Shi, ${ }^{1}$ Zhengyou Liu, ${ }^{1, *}$ and Ping Sheng ${ }^{2}$ \\ ${ }^{1}$ Key Laboratory of Artificial Micro- and Nano-structures of Ministry of Education and Department of Physics, \\ Wuhan University, Wuhan 430072, China \\ ${ }^{2}$ Department of Physics, Hong Kong University of Science and Technology, Clear Water Bay, Kowloon, Hong Kong, China
}

(Received 10 February 2010; revised manuscript received 31 May 2010; published 2 August 2010)

\begin{abstract}
We theoretically demonstrate a classical analog of electromagnetically induced transparency (EIT) in an acoustic structure. Each unit in the proposed structure consists of two square polymethyl methacrylate (PMMA) rods with one rod rotating $45^{\circ}$ relative to the other. Both square rods can support certain surface resonant modes respectively, which have almost identical resonant frequencies but highly different quality factors. Thus, the two resonant modes can act as radiative mode and dark mode in the analog, respectively, and the destructive interference of them results in EIT-like transmission effect in our structure.
\end{abstract}

DOI: 10.1103/PhysRevE.82.026601 PACS number(s): 43.20.+g, 43.35. $+\mathrm{d}, 43.40 .+\mathrm{s}$, 42.50.Gy

Electromagnetically induced transparency is the phenomenon that a sharp transparent window associated with steep dispersion is induced into opaque atomic mediums $[1,2]$. It is due to the quantum destructive interference of the different excitation pathways to the upper level in three-level atomic systems. The intriguing properties of EIT have been utilized to reduce the group velocity of light [3] and freeze light [4,5]. Recently, classical analogs of EIT [6] have been demonstrated in a waveguide side coupled to optical resonators [7-13] or in metamaterials [14-19]. Especially, mimicking EIT-like phenomenon in metamaterials has attracted much attention for it might pave the way for coherent controlling electromagnetic wave in more compact devices. However, so far there have been few works [20] related to acoustic analogs of EIT, although acoustic wave is also one kind of classical waves. We believe that, similar to electromagnetic wave, EIT-like behavior might find applications in acoustic wave.

In this work, an acoustic structure is proposed to exhibit a phenomenon with EIT-like characteristics. Each of the units in the structure consists of two square rods with one rod rotating $45^{\circ}$ relative to the other. The square rods can support surface resonant modes propagating around the circumference of them [21]. These resonant modes can couple to incident waves from free space and thus their losses are predominately due to radiation coupling, for the absorption in such acoustic system is neglectable. Finite-difference timedomain method (FDTD) [22] is used to analyze these resonant modes. It shows that, two certain resonant modes supported by the two rods respectively have close resonant frequencies but very different displacement field distributions. For the displacement field distributions determine the degree of the coupling between the resonant mode and the incident wave, the two resonant modes have very different quality factors. Consequently, after tuning the resonant frequencies of the two resonant modes to be identical, they can respectively act in the analog as radiative mode and dark mode, and the destructive interference of them results in the

\footnotetext{
*Author to whom all correspondence should be addressed; zyliu@whu.edu.cn
}

EIT-like transmission response. In addition, an analytical model involving two coupled resonators is used to corroborate the simulation.

The FDTD method for acoustic wave is developed from the two-dimensional elastic equations [23],

$$
\begin{gathered}
\rho \frac{\partial^{2} u_{x}}{\partial t^{2}}=\frac{\partial \tau_{x x}}{\partial x}+\frac{\partial \tau_{x y}}{\partial y}, \quad \rho \frac{\partial^{2} u_{y}}{\partial t^{2}}=\frac{\partial \tau_{x y}}{\partial x}+\frac{\partial \tau_{y y}}{\partial y}, \\
\tau_{x x}=(\lambda+2 \mu) \frac{\partial u_{x}}{\partial x}+\lambda \frac{\partial u_{y}}{\partial y}, \\
\tau_{y y}=(\lambda+2 \mu) \frac{\partial u_{y}}{\partial y}+\lambda \frac{\partial u_{x}}{\partial x}, \\
\tau_{x y}=\mu\left(\frac{\partial u_{x}}{\partial y}+\frac{\partial u_{y}}{\partial x}\right),
\end{gathered}
$$

where $u_{i}(i=x, y)$ is the displacement, $\tau_{i j}(i, j=x, y)$ is the stress (for acoustic wave, it can be simplified as pressure $P$ and the familiar acoustic wave equation based on variable $P$ can be derived from Eqs. (1) and (2), $\lambda$ and $\mu$ are the Lame coefficients, and $\rho$ is the density. After discretization of Eqs. (1) and (2) in both the spatial and time domains, the differential equations are transferred into difference equations. The specific forms of the discretized elastic equations, which are exploited in the FDTD method, can be found in Refs. $[24,25]$.

To begin with, we analyze at first the eigenmodes supported by a single square PMMA $\left(\rho=1000 \mathrm{~kg} / \mathrm{m}^{3}, c_{l}\right.$ $=2700 \mathrm{~m} / \mathrm{s}$, and $\left.c_{t}=1102 \mathrm{~m} / \mathrm{s}\right)$ rod immersed in water, for they will provide the fundamental modes to mimic EIT as shown later. Figures 1(a) and 1(b) demonstrate respectively the displacement vector fields of the eigen modes at frequencies of $0.89399(2 \pi v / a$ ) and $0.90989(2 \pi v / a$ ) (where $\mathrm{v}$ is velocity of acoustic wave in water, and $a=b / 0.6$ with $b$ being the side length of the square rod and $a$ being the lattice constant of the periodic rod array which will be used below), supported by the single rod. As can be seen, at each frequency, there are two symmetry related degenerate modes. Figures 1(a) and 1(b) are presented by the intention that the 


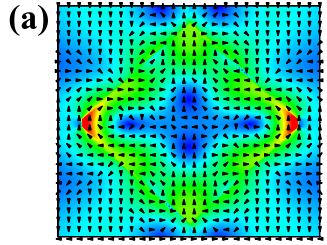

(b)
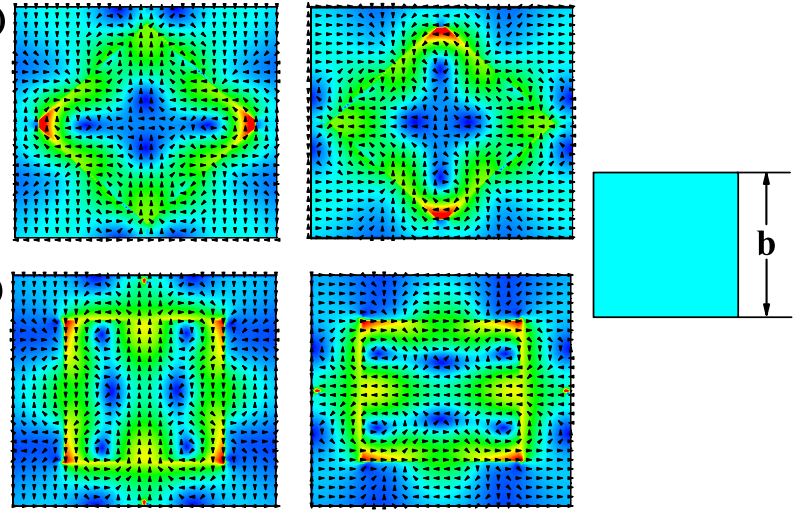

FIG. 1. (Color online) The displacement vector fields of the eigen modes supported by a single rod. (a) The two degenerate modes at frequency 0.89399 , which, for the convenience of comparing with Fig. 2(a), are presented with a clockwise rotation by $45^{\circ}$ (b) The two degenerate modes at frequency 0.90989 . The side length of the square rod is denoted by $b$. Brightness represents the magnitude of the displacement vector and black arrows denote its directions.

left panels show the modes easily excited by verticalincidence waves, while the right panels show the modes easily excited by horizontal-incidence waves and for the convenience for the later-on description. For the purposes, the degenerate modes in Fig. 1(a) have both been rotated clockwise by $45^{\circ}$, in order to align with the waves' incident directions. To be specific, we will use the two modes shown at the left panel to mimic EIT in below, which thus means a vertical incidence is assumed. From Fig. 1, we can also see that the intense fields locate on the water-PMMA interfaces around the rod, which demonstrates the surface wave characteristic of the eigenmodes.

To tune the eigenfrequencies of the two eigenmodes to be identical and also to seek the simulation convenience, we arrange the rods in the form of periodic arrays, in which the interaction of the eigenmode of each single rod will moderately adjust the eigenfrequency. Thus, via proper selection of the geometric parameters, the eigenfrequencies of the two eigenmodes can be tuned to be almost identical. Here, the rods are arranged to form the periodic array $\mathbf{A}$ and periodic array $\mathbf{B}$, which are shown in Figs. 2(a) and 2(b) respectively. For the purpose of exciting different resonant mode in the two arrays respectively, the rods of the periodic array $\mathbf{A}$ have been rotated $45^{\circ}$. As normal-incidence waves illuminate upon each rod array alone, the resonant mode can be excited, which results in scattering losses and the emergence of transmission dips in the spectra. In Fig. 2, the transmission spectra of (a) the rod array $\mathbf{A}$ alone and (b) the rod array $\mathbf{B}$ alone are shown. In both figures, the transmission spectra exhibit the transmission dips center around almost identical frequencies. However, the two resonant modes differ in the quality factors $Q_{\mathbf{A}, \mathbf{B}}=\frac{\omega_{\mathbf{A}, \mathbf{B}}}{\Delta \omega_{\mathbf{A}, \mathbf{B}}}$ (here $\omega_{\mathbf{A}, \mathbf{B}}$ are the resonant frequencies, $\Delta \omega_{\mathbf{A}, \mathbf{B}}$ are the bandwidths, and they can be obtained via the transmission spectra in Fig. 2) about an order of magnitude $\left(Q_{\mathbf{A}}=13.3\right.$ and $\left.Q_{\mathbf{B}}=290.1\right)$, which implies they couple to incidence waves very differently. To explain this difference, Fig. 2 displays the displacement vector fields of the two reso- (a)
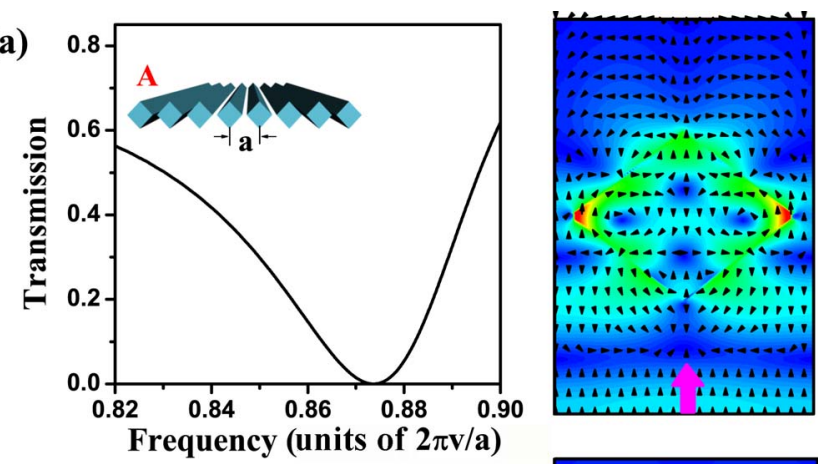

(b)
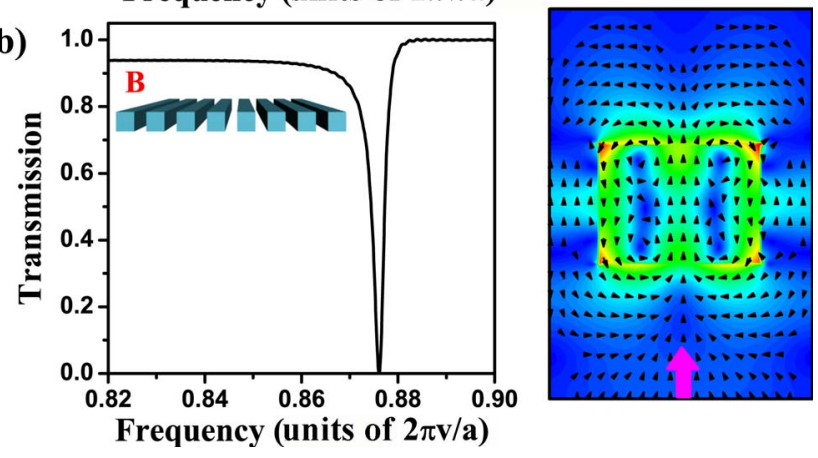

FIG. 2. (Color online) (a) Transmission spectrum for the periodic rod array $\mathbf{A}$ alone and the displacement vector field of the corresponding resonant mode. (b) Same as (a) for the periodic rod array $\mathbf{B}$ alone. In both figures: $a$ is the lattice constant. $a=b / 0.6$ is set for both arrays. Brightness represents the magnitude of the displacement vector and black arrows denote its directions. Magenta arrows indicate the incident direction.

nant modes which are similar to the displacement vector eigenfields shown in the left panel of Fig. 1. However, as can be seen, most of the displacement vectors near the incident end of the rod have vertical components in Fig. 2(a). On the contrary, in Fig. 2(b), most of the displacement vectors near the corresponding position point to the horizontal direction while only the central ones point to vertical direction. For the normal-incidence wave is longitudinal wave in water and its displacement vectors are along vertical direction, it is obvious that the displacement vectors in Fig. 2(a) can couple to the incidence wave much easier. Therefore, in the acoustic analog of EIT, the resonant mode of the periodic rod array $\mathbf{A}$ will act as a radiative mode for its lower quality factor while the resonant mode of the periodic rod array $\mathbf{B}$ will act as a dark mode for its much higher quality factor.

Then, as the two separate periodic rod arrays are combined into a single structure, EIT-like phenomenon can be observed. In Fig. 3(a), the transmission spectra of the proposed acoustic structure for different separations are presented. In all these transmission spectra (red solid lines), narrow transparency peaks appear at the center of the broad transmission dips. With the increase of the separations, the coupling strength between the radiative mode and the dark mode successively decreases. As a result, the transparency peaks keep decreasing and become narrower, which is similar to the quantum EIT in an atomic system. To better comprehend the transmission response of the structure, we have introduced a simple model with two coupled resonators $[5,15]$. The periodic rod array $\mathbf{A}$ is represented by resonator 


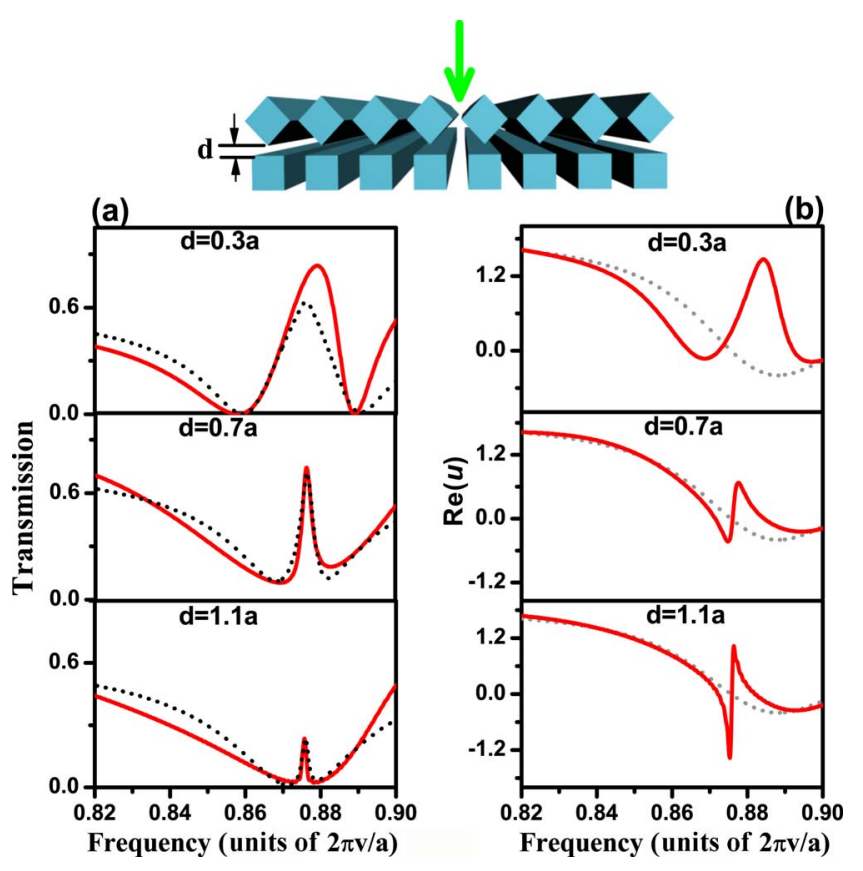

FIG. 3. (Color online) (a) Transmission spectra of the proposed structure for different separations $d$. Solid red lines represent the results obtained via the simulation; dotted black lines represent the results obtained via the analytical formula. (b) Dispersive responses of the structures. $u$ is the displacement in the center between the two rod arrays. Dotted gray lines represent the dispersive responses of rod $\mathbf{A}$ array alone as a comparison.

1 , which is subject to incidence waves $I(t)=e^{i \omega t}$. The periodic rod array $\mathbf{B}$ is represented by resonator 2 , which can be excited by the couple between the two resonators. The characteristic quantities $x_{1}(t)=a(\omega) e^{i \omega t}$ and $x_{2}(t)=b(\omega) e^{i \omega t}$, which describe the resonant states of resonators 1 and 2 , satisfy the coupled equations,

$$
\begin{gathered}
\ddot{x}_{1}(t)+\gamma_{1} \dot{x}_{1}(t)+\omega_{0}^{2} x_{1}(t)-\kappa x_{2}(t)=I(t), \\
\ddot{x}_{2}(t)+\gamma_{2} \dot{x}_{2}(t)+\left(\omega_{0}+\delta\right)^{2} x_{2}(t)-\kappa x_{1}(t)=0 .
\end{gathered}
$$

Here $\omega_{0}$ is the resonant frequency of resonator $1 . \delta$ is the detuning of the resonant frequency between resonator 2 and resonator $1\left(\delta \ll \gamma_{1}\right), \gamma_{1}$ and $\gamma_{2}$ are the losses in resonators 1 and 2, respectively, $\left(\gamma_{2} \ll \gamma_{1} \ll \omega_{0}\right)$. These losses are predominantly due to scattering losses, for the absorption in such acoustic system is neglectable. $\kappa$ is the coupling coefficient of the two resonators. After substituting $x_{1}(t)=a(\omega) e^{i \omega t}$, $x_{2}(t)=b(\omega) e^{i \omega t}$ in Eqs. (3) and (4), the amplitude of both characteristic quantities can be described by the equations,

$$
\begin{gathered}
a\left(\omega_{0}^{2}-\omega^{2}+i \omega \gamma_{1}\right)-\kappa b=1, \\
b\left[\left(\omega_{0}+\delta\right)^{2}-\omega^{2}+i \omega \gamma_{2}\right]-\kappa a=0 .
\end{gathered}
$$

By solving Eqs. (5) and (6), we can obtain

$$
x_{1}(t)=\frac{\left(\omega_{0}+\delta\right)^{2}-\omega^{2}+i \omega \gamma_{2}}{\left(\omega_{0}^{2}-\omega^{2}+i \omega \gamma_{1}\right)\left[\left(\omega_{0}+\delta\right)^{2}-\omega^{2}+i \omega \gamma_{2}\right]-\kappa^{2}} e^{i \omega t}
$$

Because resonator 1 couples with incidence waves $I(t)$, and the energy scattered from this resonator is dominant, thus the energy scattered from the coupled resonators can be presented as

$$
P_{s}(t)=I(t) \dot{x}_{1}(t)
$$

With the approximation $\omega-\omega_{0} \ll \omega_{0}$ and therefore $\omega_{0}^{2}-\omega^{2}$ $\approx 2 \omega\left(\omega_{0}-\omega\right)$, the scattered energy $P_{s}(\omega)$ during one period of oscillation of incidence waves $I(t)$ can be found

$$
P_{s}(\omega)=\frac{i}{2} \frac{\left(\omega_{0}+\delta-\omega\right)+i \frac{\gamma_{2}}{2}}{\left(\omega_{0}-\omega+i \frac{\gamma_{1}}{2}\right)\left(\omega_{0}+\delta-\omega+i \frac{\gamma_{2}}{2}\right)-\frac{\kappa^{2}}{4}}
$$

In the processing procedure, $\kappa$ is a fitting parameter, so we still employ $\kappa$ to represent $\frac{\kappa}{\omega}$. Here, the proposed structure is an infinite periodic structure, and the corresponding wavelength of the incidence wave is larger than the lattice constant $a$. Thus, for normal-incidence plane waves, only 0 -order diffraction needs to be considered, and scattered wave is in the normal direction. Moreover, as shown in Fig. 2 , the incidence wave is backward scattered by the resonant mode, therefore the reflection $\mathrm{R}$ can be replaced by $P_{s}(\omega)$. Consequently, the transmission spectra can be obtained as $T(\omega)=1-P_{s}(\omega)$. The results calculated by the analytical model are also presented in Fig. 3(a) (dotted black lines). The parameters needed in the analytical calculation can be obtained via the transmission spectra of each rod array (Fig. 2). Here, $\omega_{0}=0.87369(2 \pi v / a), \quad \delta=0.0024(2 \pi v / a), \quad \gamma_{1}$ $=0.03278(2 \pi v / a), \gamma_{2}=0.00151(2 \pi v / a)$, and $\kappa$ is the fitting parameter. As can be seen, the simulated results are reproduced well by the analytical results except the very close separation cases. For these close separation cases, the coupling strength between the radiative mode and the dark mode is so strong that the resonant frequency of each mode is modified. Thus, the simulated transparency peaks deviates from the analytical peaks which have been calculated using the unchanged parameters. While in the long separation cases, weak coupling has not altered the resonant frequency of each mode, and thus simulated peaks fit the analytical peaks very well. In both close and long separation situations, the asymmetric line shape of each resonant mode has also introduced minor discrepancy outside the regions of the transparency peaks, for the line shape of each resonant mode should be symmetry in the coupled resonators model. Anyway, the coupled resonators model has been good enough for us to give a quantitative description of our system. Beside the narrow transparency peak, a highly dispersive behavior is also an important feature of EIT. In Fig. 3(b), the dispersive responses of the structures are shown (red solid lines). These dispersive responses can be obtained via Fourier spectra of the real part of the displacements in the center between the two rod arrays [13]. The dispersions are normal and have 

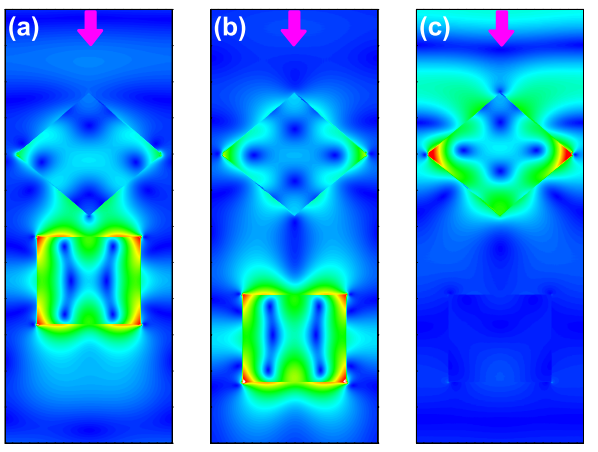

FIG. 4. (Color online) (a) The displacement field distribution at the transparency peak frequency $\omega=0.879(2 \pi v / a)$ of the structure with separation $d=0.3 a$. (b) The field distribution at the transparency peak frequency $\omega=0.875(2 \pi v / a)$ of the structure with separation $d=1.1 a$. (c) The field distribution at the frequency $\omega$ $=0.86(2 \pi v / a)$ of the same structure as in (b). Magenta arrows indicate the incident direction.

very steep slope, which indicates that significant reduction of group velocity of acoustic wave can be achieved.

At last, to visualize and further understand the EITbehavior of our structure, the displacement field distributions are demonstrated. In Fig. 4(a), the field distribution at the transparency peak frequency $\omega=0.879(2 \pi v / a)$ of the structure with separation $d=0.3 a$ is shown. Both radiative mode and dark mode are excited, and strong coupling between the two modes can clearly be seen. Due to the destructive interference, the radiative mode is suppressed and thus with much weaker intensity. Comparatively, for the field distribution at the transparency peak frequency $\omega=0.875(2 \pi v / a)$ of the structure with separation $d=1.1 \mathrm{a}$ [Fig. 4(b)], almost identical field distribution can be seen. However, in this case, the coupling between the two modes is much weaker. This confirms our aforementioned judgment that, with the longer separations, the analytical peaks are more consistent with the simulated peaks because of the weaker coupling. In addition, in Fig. 4(c), we show the field distribution at the frequency $\omega$ $=0.86(2 \pi v / a)$ of the structure with separation $d=1.1 a$. For this frequency deviates from the resonant frequency of the dark mode, only radiative mode is strongly excited and incident plane waves are backward scattered. By comprehensive consideration of all the field distributions, we are able to provide a clear description of the EIT-like behavior of our structure. As the plane acoustic wave at the resonant frequency is incident on the structure, the radiative mode is excited in the first place. And then, the dark mode is excited via the scattered waves of the radiative mode. Thus, there are two possible excitation pathways, namely, the direct scattering from the radiative mode and the indirect scattering from the dark mode via the forward and backward coupling between the two modes. In a three-level atomic system (composed of a ground state $|0\rangle$, a metastable state $|1\rangle$ and an upper state $|2\rangle)$, the probe laser drives the transition $|0\rangle$ $-|2\rangle$ and the pump laser drives the transition $|1\rangle-|2\rangle$. For $|0\rangle-|1\rangle$ is a dipole-forbidden transition, there are only two possible pathways, namely, $|0\rangle-|2\rangle$ and $|0\rangle-|2\rangle-|1\rangle-|2\rangle$, and the destructive interference of these two pathways results in EIT [1]. Obviously, there is a direct analogy between our system and the three-level atomic EIT system. The direct scattering from the radiative mode is in analogy with the possible pathway $|0\rangle-|2\rangle$ while the indirect scattering from the dark mode via the forward and backward coupling between the two modes corresponds to the possible pathway $|0\rangle-|2\rangle-|1\rangle-|2\rangle$. Therefore, the destructive interference between the direct and the indirect excitation pathways results in EIT-like behavior in our system.

In conclusion, we have shown that an acoustic structure composed of two different square rod arrays could exhibit the EIT-like phenomenon. With proper geometrical arrangement, individual rod in each periodic array can support certain resonant modes, which have almost identical resonant frequencies but very different quality factors. Thus, these modes can act as radiative mode and dark mode respectively in the EIT mimicking. Both simulation and analytical model are exploited to study the transmission response of the structure, and narrow transparency peaks and highly dispersive behaviors have been observed. Since the structure is scaleable, the acoustic EIT-behavior can be achieved at a broad range of frequencies.

\section{ACKNOWLEDGMENTS}

This work was supported by NSFC/RGC joint research Grant Nos. 10731160613 and N_HKUST632/07, NSFC Grant Nos. 10874131, 50425206, and 10974147, and National Science Fund for Talent Training in Basic Science (Grant No. J0830310).
[1] S. E. Harris, Phys. Today 50, 36 (1997).

[2] M. Fleischhauer, A. Imamoglu, and J. P. Marangos, Rev. Mod. Phys. 77, 633 (2005).

[3] L. V. Hau, S. E. Harris, Z. Dutton, and C. H. Behroozi, Nature (London) 397, 594 (1999).

[4] C. Liu, Z. Dutton, C. H. Behroozi, and L. V. Hau, Nature (London) 409, 490 (2001).

[5] M. Bajcsy, A. S. Zibrov, and M. D. Lukin, Nature (London) 426, 638 (2003).

[6] C. L. Garrido Alzar, M. A. G. Martinez, and P. Nussenzveig,
Am. J. Phys. 70, 37 (2002).

[7] L. Maleki, A. B. Matsko, A. A. Savchenkov, and V. S. Ilchenko, Opt. Lett. 29, 626 (2004).

[8] D. D. Smith, H. Chang, K. A. Fuller, A. T. Rosenberger, and R. W. Boyd, Phys. Rev. A 69, 063804 (2004).

[9] M. F. Yanik, W. Suh, Z. Wang, and S. Fan, Phys. Rev. Lett. 93, 233903 (2004).

[10] Q. Xu, S. Sandhu, M. L. Povinelli, J. Shakya, S. Fan, and M. Lipson, Phys. Rev. Lett. 96, 123901 (2006).

[11] K. Totsuka, N. Kobayashi, and M. Tomita, Phys. Rev. Lett. 98, 
213904 (2007).

[12] X. Yang, M. Yu, D.-L. Kwong, and C. W. Wong, Phys. Rev. Lett. 102, 173902 (2009).

[13] C. H. Raymond Ooi and C. H. Kam, Phys. Rev. B 81, 195119 (2010).

[14] N. Papasimakis, V. A. Fedotov, N. I. Zheludev, and S. L. Prosvirnin, Phys. Rev. Lett. 101, 253903 (2008).

[15] S. Zhang, D. A. Genov, Y. Wang, M. Liu, and X. Zhang, Phys. Rev. Lett. 101, 047401 (2008).

[16] S.-Y. Chiam, R. Singh, C. Rockstuhl, F. Lederer, W. Zhang, and A. A. Bettiol, Phys. Rev. B 80, 153103 (2009).

[17] N. Liu, L. Langguth, T. Weiss, J. Kastel, M. Fleischhauer, T. Pfau, and H. Giessen, Nature Mater. 8, 758 (2009).

[18] P. Tassin, L. Zhang, T. Koschny, E. N. Economou, and C. M. Soukoulis, Phys. Rev. Lett. 102, 053901 (2009).
[19] V. Yannopapas, E. Paspalakis, and N. V. Vitanov, Phys. Rev. B 80, 035104 (2009).

[20] E. H. El Boudouti, T. Mrabti, H. Al-Wahsh, B. DjafariRouhani, A. Akjouj, and L. Dobrzynski, J. Phys.: Condens. Matter 20, 255212 (2008).

[21] A. Viktorov, Rayleigh and Lamb Waves: Physical Theory and Applications (Plenum Press, New York, 1967).

[22] A. Taflove, Computational Electrodynamics: The FiniteDifference Time-Domain Method (Artech House INC, Norwood, 2000).

[23] L. D. Landau and E. M. Lifshitz, Theory of Elasticity (Pergamon, London, 1959).

[24] M. M. Sigalas and N. Garcia, J. Appl. Phys. 87, 3122 (2000).

[25] P.-F. Hsieh, T.-T. Wu, and J.-H. Sun, IEEE Trans. Ultrason. Ferroelectr. Freq. Control 53, 148 (2006). 